\title{
Explanations for the Pursuit of Exercise
}

Psychology 221-01

Paper Assignment--Fall 2015

Written by: Clark Xu

Evaluated by: Prof. Holloway 
Contemporary culture in the United States has recently placed the spotlight on physical exercise as a solution to emerging public health issues of obesity, heart disease, and stroke. However, fascination with exercise stems from even deeper cultural roots. In Plato's Republic, Socrates argues that a virtuous soul motivates exercise for maintaining a beautiful, athletic body. Similarly, Cicero suggests in his essay On Old Age that youth and vitality are the source of exercise, promoting happiness and intelligence. John Locke, closer to the present-day, concurs in Some Thoughts Concerning Education that exercise prepares intellectual activity. All argue that regular exercise is more beneficial than accessible; in fact, they assume that maintaining an exercise routine is a matter of innate talent. It is time for an update. Psychological subdisciplines are a promising source of explanations for the development and maintenance of exercise habits in some humans and not others. I will interpret several experiments on exercise using the behavioral mechanism of opponent processes, neurobiological mechanism of brain regional activity, socio-cognitive mechanism of self-efficacy, and behavioral mechanism of incentive motivation.

The experimental method for the study of exercise involves human participants who perform an exercise test, while monitored for biological and cognitive measures. To explain exercise routines, researchers collect data in pre-test, test, and post-test stages. Studies with short post-test stages, ranging from five to fifteen minutes after the exercise test, suggest that individuals with different exercise lifestyles respond with different moods and valences (Blanchard et al., 2002, p.1391; Hallgren et al., 2010, p.632; Magnan et al., 2013, p.6). The findings demonstrate that active individuals experience greater increases in mood following an exercise routine, and tend to rate the routine with more positive valence, compared to sedentary individuals. By contrast, studies with long post-test stages, ranging from one to fourteen days after the exercise test, have not established that repeated exercise sessions change the valence of exercise (Strohacker et al., 2014, p.96; Walter et al., 2013, p.8). The null hypothesis is partly due to poor design, such as participant absenteeism or uncontrolled environments, leading to a lacuna in the literature. Animal research may be required to study the effects of exercise during long post-test stages, because these populations can be held captive in a controlled environment.

Data from pre-test and test stages support observations in the short post-test stage about differences in mood and valence between individuals with different exercise lifestyles. Researchers use data from the pre-test stage as a baseline for biological and cognitive measures during the test (Blanchard et al., 2002, p.1384; Magnan et al., 2013, p.4; Seymour et al., 2005, p.1235). Active individuals report over the course of the test that the exercise routine increases in 
positive valence, alleviates perceived fatigue, and temporarily lowers perceived tranquility. In reverse, sedentary individuals report lower levels of positive valence, higher levels of perceived fatigue, and greater dips in perceived tranquility for the same exercise routine (Hallgren et al., 2010, p.633; Magnan et al., 2013, p.14).

The opponent-process theory offers an explanation for these results. Exercise routines initiate a primary hedonic process, where the physiological stress of exercise stimulates negative valence, followed by an opponent hedonic process, where the bodily response to physiological stress stimulates positive valence (Solomon, 1980, p.695). Since sedentary individuals experience exercise routines as novel stimuli, the opponent process has small magnitude and long delay. The sum of both processes predicts that exercise stimulates negative valence followed by an equal amount of positive valence. Meanwhile, active individuals experience exercise routines as habituated stimuli, so that the opponent process has large magnitude and short delay. The sum predicts that exercise stimulates negative valence followed by a greater amount of positive valence. In active individuals, the stronger opponent process is the mechanism for improved mood and valence following exercise. It is elicited by appetitive conditional stimuli, called relief stimuli, which are presented a short time before an aversive unconditional stimulus (Andreatta et al., 2015, p.12). Consequently, routine lifestyles, visiting the gym or wearing a sports outfit, perpetuate exercise behavior by conditioning relief stimuli that make exercise more enjoyable for active individuals.

This explanation has a neurobiological basis if we interpret exercise as pain, and the termination of exercise as relief from pain. In the presence of an aversive unconditional stimulus, activation of the orbitofrontal cortex for decision-making follows the opposite pattern of the substantia nigra for reward (Seymour et al., 2015, p.1236). Furthermore, cerebral blood flow following electric shock with the relief stimulus is uniphasic like the response to habituated stimuli, and without the relief stimulus is biphasic like the response to novel stimuli. This supports the prediction that relief stimuli elicit the opponent process in active individuals.

Besides opponent-process theory, self-efficacy explains that active individuals are sensitive to feelings of agency and exercise to be in control, while sedentary individuals are insensitive to agency and thus have less motivation to exercise (Blanchard et al., 2002, p.1380; Hallgren et al., 2010, p.635; Magnan et al., 2013, p.6). However, self-efficacy fails to explain why the same individual might oscillate between active and sedentary lifestyles (Walter et al., 2013, p.5). On the other hand, incentive motivation is not subject to the same criticism, because reduced rates of exercise after an exercise intervention program are interpreted as extinction 
trials once exercise is no longer reinforced (Strohacker et al., 2014, p.96). Unfortunately for incentive motivation, differences between active and sedentary individuals during the absence of reinforcement are not weakened, but actually strengthened through the conditioning of relief stimuli.

If the opponent-process theory remains the best explanation for the pursuit of exercise, then practicing exercise routines is crucial to developing an active lifestyle. An individual seeking to make the transition from a sedentary to an active lifestyle should keep in mind that the emotional benefits of exercise will increase over time. Once established, exercise routines are self-perpetuating, as long as the individual follows the same routine. A different routine, however, will eliminate the relief stimuli and reset the opponent process, threatening recently active individuals with the risk of relapse. Although it is neither easy nor impossible to set up an exercise routine, individuals who understand the behavioral mechanisms responsible for the emotional valence of exercise have already begun to secure their final success. 


\section{$\underline{\text { Annotated Bibliography }}$}

Andreatta, M., Muhlberger, A., \& Pauli, P. (2015). When Does Pleasure Start After the End of Pain? The Time Course of Relief. The Journal of Comparative Neurology, 0(1), 1-15. Retrieved October 19, 2015, from http://onlinelibrary.wiley.com/

Previous studies suggest that backwards conditioning of CS with aversive US will allow the CS to become appetitive and to elicit the b-process, or relief CR, when presented some time after the US. This study featured human volunteers given either fear conditioning, relief conditioning, or no conditioning, where the US was an electric shock rated for pain on a visual-analog-scale based on a threshold procedure, the fear CS was a white noise delivered before US, the relief CS was yellow shapes on a black background, and the $\mathrm{UR} / \mathrm{CR}$ was the startle response on an EMG and ratings of emotional valence on a visual-analog-scale. During test, the fear CS and relief CS were presented in the absence of the US. The study concludes that relief CS was aversive at no delay from US with startle and negative valence, relief CS was neutral at threesecond delay from US, and relief CS was appetitive at six-second delay from US with startle attenuation and positive valence, confirming that relief CS best elicits relief CR in humans given a short delay from aversive US.

Blanchard, C., Rodgers, W., Courneya, K., \& Spence, J. (2002). Moderators of the Exercise/Feeling-State Relationship: The Influence of Self-Efficacy, Baseline, and InTask Feeling States at Moderate- and High-Intensity Exercise. Journal of Applied Social Psychology, 32(7), 1379-1395. Retrieved October 21, 2015, from http://onlinelibrary.wiley.com/

The study examines whether self-efficacy during moderate to high intensity exercise is the mechanism for positive correlation between acute exercise and positive valence, and negative correlation between acute exercise and anxiety. This study featured female human volunteers assigned no exercise, $50 \%$ HRR of 15 minutes, $50 \%$ HRR of 30 minutes, and $85 \%$ HRR of 30 minutes, where HRR was measured by difference between pre-exercise measurement of resting heart rate and during-exercise measurement of active heart rate on a cycle ergometer, subjective feeling of acute exercise was measured by Ratings of Perceived Exertion scale, self-efficacy was measured by $0-100 \%$ confidence rating of participant's own ability to complete the task, and emotional valence was measured by Exercise-Induced Feeling Inventory. During test, all measurements were taken at $0 \% \mathrm{HRR}$ before, every 15 minutes during, and at $0 \% \mathrm{HRR}$ after exercise. The study concludes for the sample that self-efficacy did not influence any factors except for a temporary drop in tranquility during exercise at $85 \% \mathrm{HRR}$, a temporary rise in physical exhaustion during exercise at $85 \% \mathrm{HRR}$, and steady increases in revitalization and positive through all stages for $50 \% \mathrm{HRR}$ and $85 \% \mathrm{HRR}$.

Hallgren, M., Moss, N., \& Gastin, P. (2010). Regular exercise participation mediates the affective response to acute bouts of vigorous exercise. Journal of Sports Science and Medicine, 9(1), 629-637. Retrieved October 21, 2015, from http://www.jssm.org/

The study suggests that inconsistent findings in the literature, where some studies conclude that exercise habits do not influence mood changes after acute exercise, while other studies conclude that low exercise improves the mood of all participants and moderate exercise only improves the mood of active participants, could be explained by considering exercise intensity. This study featured university volunteers categorized as regular exerciser or non-regular exerciser by a pre-study questionnaire, and administered a questionnaire composed of the Exercise-Induced Feeling Inventory, Profile of Mood States, and State-Trait Anxiety Inventory, at baseline, before test, after test ten minutes, and after test twenty-five minutes. During test, participants pedaled a cycle ergometer to exhaustion, with increasing workload every two minutes. The study concludes that regular exercisers experienced on the EFI steady increases in positive engagement, tranquility, and revitalization, or steady decreases in physical exhaustion, as well as on the POMS steady decreases in anger, depression, tension, confusion, and fatigue or steady increases in vigor; non-regular exercisers experienced on the EFI a temporary fall at after test ten minutes in positive engagement, tranquility, and revitalization, or a temporary rise at after test ten minutes in physical exhaustion, as well as 


\section{Explanations for the Pursuit of Exercise}

Clark Xu

on the POMS a temporary rise at after test ten minutes in depression and fatigue, or a temporary fall at after test ten minutes in vigor.

Magnan, R., Kwan, B., \& Bryan, A. (2013). Effects of current physical activity on affective response to exercise: Physical and social-cognitive mechanisms. Psychology and Health, 28(4), 418-433. Retrieved October 20, 2015, from http://www.ncbi.nlm.nih.gov/pmc/

The study examines the hypothesis that active individuals will report higher levels of positive emotional valence for exercise than sedentary individuals, with possible mechanisms of BMI, VO2-max, core temperature, heart rate, and self-efficacy. This study featured undergraduate volunteers divided into an active group representative of average exercise at the university and a sedentary group reporting less than 90 minutes of weekly physical activity, where exercise behavior was measured by Physical Activity Recall, self-efficacy was measured by 7 items on 7 point scales, BMI was measured by a height and weight calculation, emotional valence was measured by Physical Affect Activity Scale, heart rate was measured by HR monitor, and core temperature was measured by infrared tympanic thermometer. During test, emotional valence, heart rate, and core temperature were measured every 10 minutes once the participant reached $65 \%$ VO2-max on a treadmill. The study concludes that active individuals experience greater increases in positive emotional valence, smaller temporary drops in tranquility, less fatigue, and less negative emotional valence over the course of 30 minutes of $65 \%$ VO2-max exercise compared to sedentary individuals.

Seymour, B., O’Doherty, J., Koltzenburg, M., Wiech, K., Frackowiak, R., Friston, K., \& Dolan, R. (2005). Opponent appetitive-aversive neural processes underlie predictive learning of pain relief. Nature Neuroscience, 8(9), 1234-1240. Retrieved October 22, 2015, from http:/www.nature.com/neuro/index.html/

Existing literature on pleasure and relief support the prediction of opponent-process theory that both are appetitive stimuli motivating the UR/CR, but neural mechanisms for pleasure and relief have not been adequately examined. This study featured healthy human volunteers first trained using a short-delay classical conditioning procedure with the US of a heating node applied to a patch of skin covered with capsaicin on the left leg, pain CS of abstract images on a screen followed by increased node temperature, relief CS of abstract images followed by decreased node temperature, and UR/CR of activity in the substantia nigra and the orbitofronal cortex as measured on fMRI when the node changed from baseline temperature. During test, the fMRI group was either exposed to the pain CS and US of temperature increase, only the US of temperature increase, the relief CS and US of temperature decrease, and only the US of temperature decrease. The study concludes that activity in the substantia nigra followed appetitive temporal difference prediction error, where CS presentation resulted in a negative parabola graph for signal change and no CS presentation resulted in a sine graph for signal change, to show that relief CS elicits an appetitive response; meanwhile, activity in the orbitofrontal cortex followed aversive temporal difference prediction error, where CS presentation resulted in a parabola graph for signal change and no CS presentation resulted in a negative sine graph for signal change, to show that pain CS elicits an aversive response.

\section{Solomon, R. (1980). The Opponent-Process Theory of Acquired Motivation. American Psychologist, 35(8), 691-712. Retrieved October 17, 2015, from http://www.apa.org/}

The article considers changes in affective contrast for ducklings when a mother is presented and removed, for human babies when a bottle is removed and returned, for parachuters when a jump is started and finished, and for dogs when an electric shock is applied and removed, to describe the standard and habituated pattern of changes to affective contrast over time. The article suggests that the standard pattern to US with few presentations is the sum of a primary hedonic process and a short, weak, and delayed opponent hedonic process, and that the habituated pattern to US with many presentations is the sum of a primary hedonic process and a long, strong, and immediate opponent hedonic process. Finally, it concludes that the primary hedonic process and opponent hedonic process may be linked to opposite emotional valences, and that both the primary hedonic process and the opponent hedonic process may be activated or modified by CS trained through repeated pairings with the US. 
Strohacker, K., Galarraga, G., \& Williams, D. (2014). The Impact of Incentives on Exercise Behavior: A Systematic Review of Randomized Controlled Trials. Annals of Behavioral Medicine, 48(1), 92-99. Retrieved October 17, 2015, from http://link.springer.com/

The study performed a meta-analysis of ten studies from a pool of 1,873 reports for the impact of schedules of operant conditioning on the amount of exercise performed by humans across various age groups. The meta-analysis found that researchers used cash or prizes to reward exercise in adults, and used television or toys to reward exercise in children, providing positive reinforcement on a fixed ratio schedule, a variable ratio schedule, or a fixed interval schedule. The study concludes that the prediction of behavioral economics, that operant conditioning for exercise can increase exercise behavior, holds true for FR and FI but not VR schedules. Meanwhile, the existing literature has not been able to study intrinsic motivation, describe extinction effects of the operant conditioning of exercise following the termination of an exercise program, or generalize the impact of operant conditioning on humans across age, sex, or weight.

Walter, K., Haaren, B., Loffler, S., Hartel, S., Jansen, C., Werner, C., ... \& Hey, S. (2013). Acute and medium term effects of a 10 -week running intervention on mood state in apprentices. Frontiers in Psychology, 4(411), 1-10. Retrieved October 21, 2015, from http://www.frontiersin.org/

To test the hypothesis that acute exercise improves general mood among young adults, this study featured 23 university volunteers that were divided into a control group given no exercise intervention and an experimental group given a 10 week exercise intervention, where initial endurance was calculated as heart rate zones based on measurements of lactate threshold from blood samples taken in a three-stage progressive running test, general mood was measured by Wilhelm-Schoebi short-scale polling for valence, calmness, and arousal five times a day plus three times after each training session, and general activity was measured by a hip-attached accelerometer. During the test, the experimental group received bi-weekly running sessions for 10 weeks that progressively increased from 30 minutes to 60 minutes and then was monitored for 2 weeks, while the control group was monitored for 10 weeks and 2 weeks. The study concludes that the experimental group engaged in higher-intensity physical activity than the control group even when intensity declined somewhat in the experimental group during the 2 week period, the experimental group reported increased arousal, decreased valence, and decreased calmness immediately after running sessions in the second week, and the experimental group reported increased arousal, valence, and calmness immediately after running sessions averaged across the second, sixth, and ninth week. The study concludes that acute exercise had short-term, but not medium-term, effects on general mood, so that general mood is impacted differently, if at all, by acute exercise in the short-term and regular exercise in the medium-term. 